\title{
EVENTOS MUSICAIS CAUSAM IMPACTOS NO MICROCLIMA DE CAVERNAS? AVALIAÇÃO DAS ALTERAÇÕES NA ATMOSFERA SUBTERRÂNEA DA GRUTA MORRO PRETO (PETAR, IPORANGA-SP)
}

\author{
Heros Augusto Santos Lobo ${ }^{1}$, José Alexandre de Jesus Perinotto ${ }^{2}$, \\ Paulo César Boggiani ${ }^{3}$ \& Silmara $\mathrm{Zago}^{4}$
}

Resumo

\begin{abstract}
O presente trabalho apresenta os resultados do monitoramento de três parâmetros atmosféricos - temperatura ambiente (T), umidade relativa do ar (UR) e dióxido de carbono $\left(\mathrm{CO}_{2}\right)$ - aferidos durante um evento musical na gruta Morro Preto, Parque Estadual Turístico do Alto Ribeira - PETAR (Iporanga-SP). A coleta e registro de dados - realizados antes, durante e depois do evento - foi realizada por meio de termohigrômetros com registradores e com sensores infra-vermelhos para $\mathrm{CO}_{2}$. Realizou-se também a contagem de pessoas presentes no dia do evento (247, no total), de forma a relacionar os dados coletados com o total de visitantes. Os dados foram analisados por meio dos seguintes procedimentos estatísticos: descrição, correlação linear e regressão linear múltipla. Os resultados e discussões evidenciam as pequenas alterações atmosféricas ocorridas no ambiente em decorrência do evento, sendo praticamente nulas para $\mathrm{T}$ e UR e mínimas para o $\mathrm{CO}_{2}$. Além disso, demonstram que as concentrações de $\mathrm{CO}_{2}$ variam também em função da dinâmica natural do ambiente - com especial destaque para a localização dos pontos de coleta e para a UR. Conclui-se que no caso específico da gruta Morro Preto, eventos musicais não causam impactos ambientais negativos à atmosfera cavernícola em função das características naturais da caverna que possibilitam troca relativamente alta de energia entre o ambiente subterrâneo e meio externo.
\end{abstract}

Palavras-chave: Espeleoturismo; Gás Carbônico; Manejo Espeleológico; Microclima.

\begin{abstract}
This paper presents the results of the monitoring of three atmospheric parameters -environmental temperature (T), relative air humidity $(\mathrm{RH})$ and carbon dioxide level $\left(\mathrm{CO}_{2}\right)$ - during a musical event realized in the Cave of Morro Preto, located in the State Touristic Park of the Upper Ribeira River (PETAR) in the district of Iporanga, São Paulo State, Brazil. Termohygrometers with dataloggers (to measure T and RH) and infrared sensors (to measure $\mathrm{CO}_{2}$ ) were used to collect the data before, during and after the musical event. The number of people present on the day of the event was also determined ( 247 total) and these data were correlated with the environmental data using descriptive statistics, linear correlation and multiple linear regression. The results show that although small alterations did occur in the environment as a consequence of the event, they were pratically null for $\mathrm{T}$ and $\mathrm{RH}$ and minimal for the level of $\mathrm{CO}_{2}$. Moreover, the $\mathrm{CO}_{2}$ concentration already varies as a function of the natural dynamics of the environment at the points of data collection. It was therefore concluded that this musical event did not cause negative environmental impacts on this cave, especially since it has a relatively high energy exchange with the external environment.
\end{abstract}

Keywords: Speleoturism; Carbon Dioxide; Speleological Management; Microclimate.

\section{INTRODUÇ̃̃O}

O uso de cavernas para a realização de eventos culturais no Brasil é bastante incomum, restrito a não mais que uma dezena com de uso religioso, como a gruta Terra Ronca em Goiás e a gruta Mangabeia em Ituaçu na Bahia.

No Estado de São Paulo, a gruta Morro Preto, situada no interior do PETAR - Parque Estadual Turístico do Alto Ribeira -, em Iporanga (figura 1) tem sido procurada para eventos musicais devido às suas características de fácil acesso e ampla abertura na entrada, o que possibilita iluminação natural, além da beleza cênica.

Diversos estudos realizados no Brasil e em outras partes do mundo abordam a questão das avaliações de impactos ambientais em cavernas e aspectos inerentes aos limites de visitação turística, com destaque para o trabalho de Cigna \& Forti (1988), com a relação dos principais impactos decorrentes do uso turístico no meio físico de uma caverna; de Hoyos et al. (1998), que contribui com os conceitos técnicos de capacidade de carga espeleoturística por meio da limitação de uso em função das variações na temperatura ambiente, bem como alertam para as conseqüências do aumento nas taxas de $\mathrm{CO}_{2}$ ambiente, podendo ativar o mecanismo de corrosão por condensação e o de Boggiani et al. (2007) para a determinação da capacidade de carga das grutas do Lago Azul e Nossa Sra. Aparecida, em Bonito-MS

O presente trabalho apresenta os resultados do monitoramento de dois atributos microclimáticos (temperatura ambiente $-\mathrm{T}-\mathrm{e}$ umidade relativa do ar - UR) e dos níveis de concentração de dióxido de

1. Turismólogo, MSc. em Geografia, Doutorando em Geociências e Meio Ambiente (UNESP - Rio Claro, SP). heroslobo@ hotmail.com 2. Geólogo, Professor Adjunto (Livre-Docente) do Depto. de Geologia Aplicada - IGCE/UNESP - Rio Claro, SP. perinoto@rc.unesp.br 3. Geólogo, Professor Doutor do Depto. de Geologia Sedimentar e Ambiental - IGc/USP - São Paulo, 


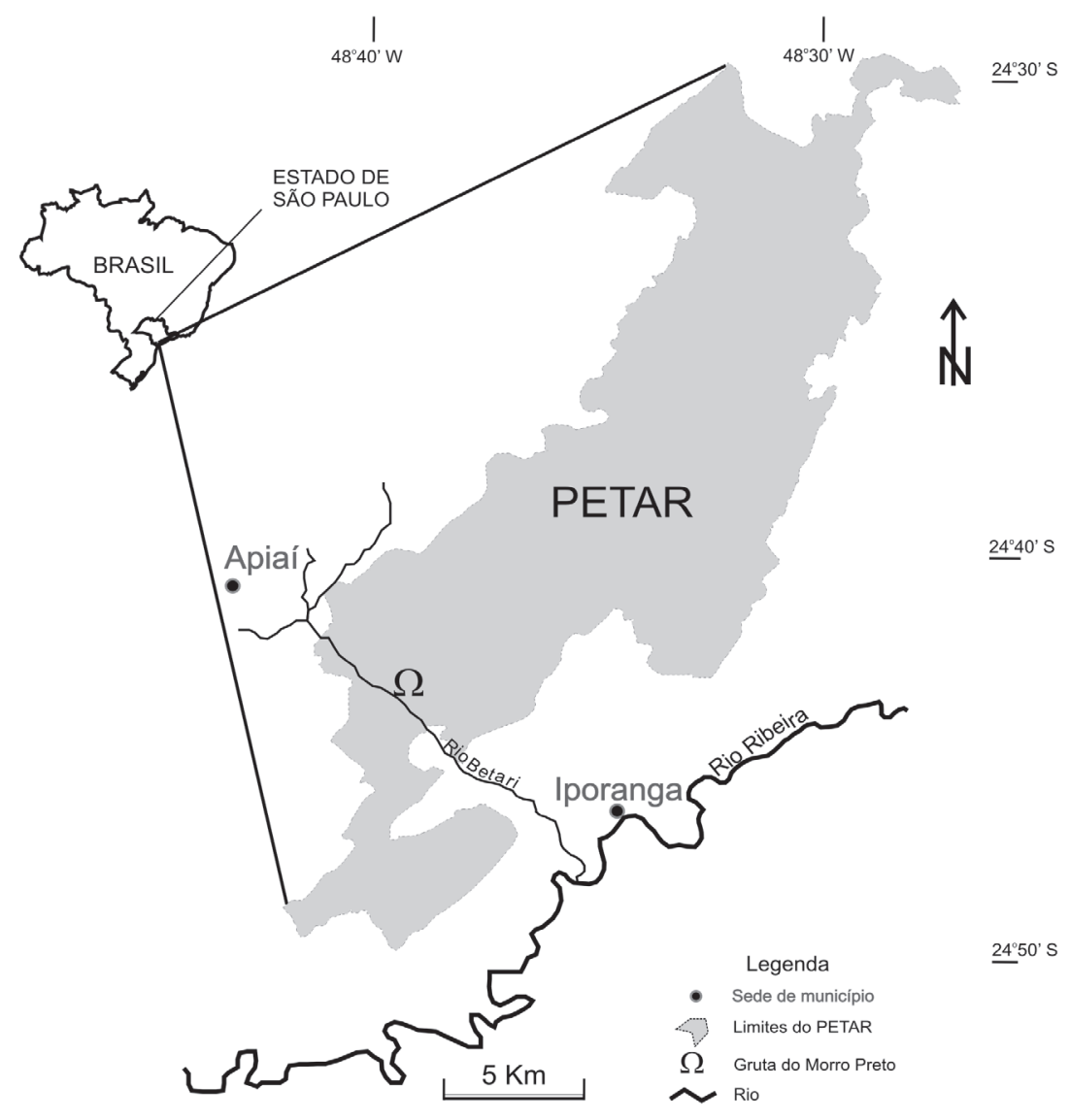

Figura 1 - Localização da Gruta do Morro Preto em relação ao PETAR. Elaborado pelos autores a partir de Karmann \& Ferrari (2002) e Lobo (2008).

carbono $-\mathrm{CO}_{2}$ - realizados durante o evento musical comemorativo aos cinqüenta anos do PETAR, no interior da gruta do Morro Preto no dia 17/05/2008. O monitoramento ambiental foi realizado antes durante e após o evento, a fim de se avaliar os impactos negativos na atmosfera cavernícola.

\section{CARACTERIZAÇÃO DA ÁREA DE ESTUDO}

A geologia da região pesquisada engloba as formações Bairro da Serra, Mina de Furnas e Passa Vinte (Karmann \& Ferrari, 2002), sendo a unidade Bairro da Serra - onde se insere a gruta do Morro Preto - a de maior expressão em área (Campanha, 1991).

A gruta corresponde à paleo-ressurgência do rio do Couto, que atualmente ressurge na gruta do Couto. Sua geomorfologia de condutos é composta basicamente por grandes salões de abatimento, fruto do avanço temporal de sua espeleogênese. Em diversos trechos de seu desenvolvimento as galerias são preenchidas parcialmente por sedimentos e blocos rochosos, aparentemente estáveis e consolidados. Comparada às outras cavernas do PETAR, a Morro Preto possui poucos espeleotemas, em sua maioria estalactites de calcita e um salão superior com flores de aragonita.

A gruta do Morro Preto possui aproximadamente
900 metros de galerias mapeadas e um circuito de visitação com quase $200 \mathrm{~m}$ de percurso. Está entre as cavernas mais visitadas do PETAR, situada no núcleo Santana do parque. O acesso a gruta é feito por uma trilha entre a vegetação nativa - floresta atlântica - e em percurso inicialmente plano e mais íngreme em sua segunda metade, com um lance de mais 150 degraus de escada para se chegar a sua entrada. A grandiosidade de suas dimensões torna a gruta semelhante a uma catedral, sendo bastante iluminada em seus dois primeiros salões.

Seus limites de visitação foram determinados em seu Plano de Ações Emergenciais, sendo de 225 visitantes/ dia em grupos de nove pessoas. Não existem estudos embasando estes valores - trata-se de uma capacidade de suporte operacional.

Devido às suas grandes dimensões e aspecto cênico, a gruta do Morro Preto tem sido tradicionalmente utilizada para as comemorações dos últimos aniversários decênios do PETAR. Também já foi utilizada nos anos setenta para a gravação de um musical, a Sinfonia do Alto Ribeira, orquestrada por Hermeto Pascoal, destacado músico brasileiro. Além disso, esporadicamente se realizam celebrações de casamentos e outras pequenas festividades locais em seu interior. 


\section{MATERIAIS E MÉTODOS}

\section{Características do evento e capacidade de carga}

O evento musical em comemoração aos cinqüenta anos do PETAR foi realizado no dia 17 de maio de 2008, com duração de uma hora (tempo total entre preparação, apresentação e dispersão dos presentes), iniciado às 15:00 h e encerrado às 16:00 h. O evento consistiu em apresentação musical de três músicos com uso de instrumentos de corda e sopro sem amplificadores e iluminação à pilha pelos presentes.

A dimensão espacial também foi considerada no aporte metodológico que subsidiou o planejamento do evento. Para tanto, restringiu-se a permanência das pessoas em duas áreas da caverna - o Salão de Entrada e o Anfiteatro principal (figura 2). Além disso, estabeleceu-se um limite de público para o evento, com base na Capacidade de Carga Física - CCF - do método de Capacidade de Carga de Cifuentes (CifuentesArias et al., 1999), a qual é expressa da forma como demonstrado na Equação 1.

$$
C C F=\frac{S}{s p} * N V
$$

Onde $S$ corresponde ao espaço total disponível, $s p$ ao espaço ocupado por cada pessoa e $N V$ o número de vezes em que o evento em questão irá ocorrer.

\section{Coleta de dados}

Os atributos microclimáticos de $\mathrm{T}$ e UR foram monitorados com o uso de termohigrômetros com registradores de dados com precisão de aproximadamente $0,2^{\circ} \mathrm{C}$ para $\mathrm{T}$ e $3 \%$ para UR.

Para o presente estudo, utilizou-se de um intervalo de tempo de um minuto entre os registros, nas dez estações de monitoramento dentro e fora da caverna, distribuídas conforme exposto na figura 2 . O experimento teve início às $17: 30$ hs do dia 16/05/2008 e encerramento às 12:27 hs do dia 18/05/2008. Este intervalo de tempo totalizou 42 horas e 57 minutos de aferição e coleta.

Embora os estudos de microclima de cavernas sejam feitos, em sua maioria, por períodos de tempo mais extensos (e.g. Pulido-Bosch et al., 1997; Hoyos et al., 1998; Boggiani et al., 2007), bons resultados também têm sido obtidos em estudos mais breves, como o trabalho de Lima \& Morais (2006) na gruta do MaquinéMG e o de Longhitano et al. (2007) na gruta ColoridaSP. Para diminuir eventuais riscos pelo curto intervalo temporal, foi implantada uma rede com dez estações de monitoramento, de modo a ampliar a abrangência espacial do estudo e permitir maior detalhamento de aspectos do microclima da gruta do Morro Preto.

A coleta de dados de $\mathrm{CO}_{2}$ foi realizada antes, durante e após o evento, com o equipamento Telaire 7001. Seu princípio de análise é através de raios infra-vermelhos, adequado para estudos ambientais. A inexistência de um registrador de dados acoplado ao equipamento impediu o monitoramento contínuo. A precisão de medição do equipamento é de $50 \mathrm{ppm}$.

Em função dos métodos adotados, foi impossível o monitoramento de $\mathrm{CO}_{2}$ em todos os pontos amostrados. Assim, optou-se pelo monitoramento de seções

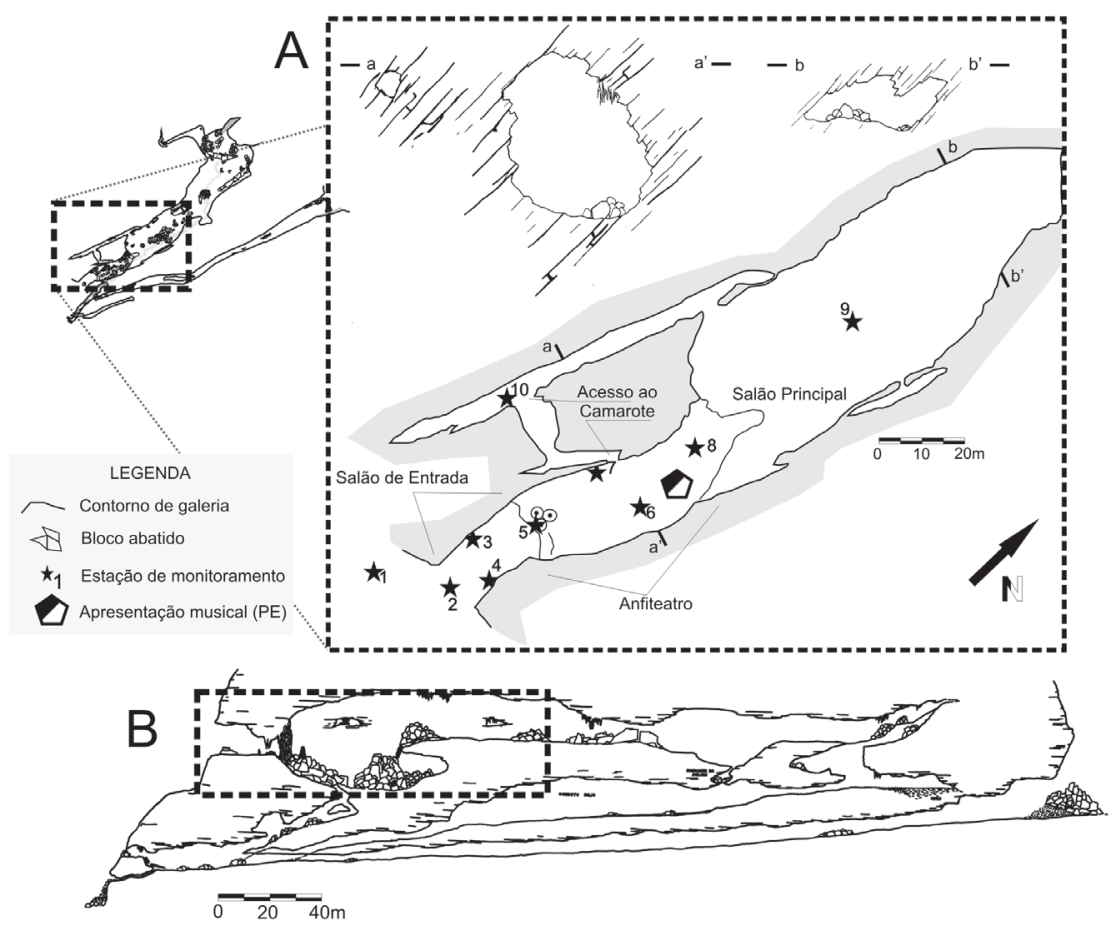

Figura 2: A - Posicionamento em planta das estações de monitoramento e do local de apresentação dos músicos na gruta do Morro Preto. B - O campo delimitado no perfil longitudinal permite a visualização do volume dos salões afetados pelo evento. Elaborada pelos autores com base em planta original de GEGEO (1998). 
amostrais nos pontos 2 (externo à caverna), 3, 5 (Salão de Entrada), 6 e 7 (Anfiteatro).

Procedimentos de análise

Os dados de T e UR foram analisados em função de sua localização espacial, buscando indícios para um provável zoneamento microclimático da Gruta do Morro Preto, por meio de gráficos isolados dos elementos e análise estatística descritiva em um primeiro momento.

Os dados coletados também foram testados por meio de testes de correlação. Para tanto, utilizou-se do coeficiente de correlação produto-momento (r) de Karl Pearson. Gerardi \& Silva (1981) explicam que o coeficiente " $r$ " indica o grau de associação linear entre duas variáveis x e y, cuja relação se expressa da forma apresentada na equação 2 .

$$
r=\frac{n \sum x_{i} y_{i}-\left(\sum x_{i}\right)\left(\sum y_{i}\right)}{\sqrt{\left[n \sum x^{2}{ }_{i}-\left(\sum x_{i}\right)^{2}\right]\left[n \sum y^{2}{ }_{i}-\left(\sum y_{i}\right)^{2}\right]}}
$$

Onde $x_{i}$ e $y_{i}$ representam os respectivos desvios de $\mathrm{x}$ e $\mathrm{y}\left(\mathrm{x}_{\mathrm{i}}-\mathrm{x}\right)$ e $\left(\mathrm{y}_{\mathrm{i}}-\mathrm{y}\right)$. O resultado para " $\mathrm{r}$ " pode variar entre +1 e -1 , dado que $r=+1$ indica uma perfeita associação positiva; $r=-1$, uma perfeita associação negativa; $r=0$, ausência de correlação (Gerardi \& Silva, 1981)

Posteriormente, conferiu-se atenção maior aos dados de temperatura. Estudos que relacionam o microclima aos impactos ambientais do turismo (e.g. Pulido-Bosch et al., 1997; Hoyos et al., 1998; Mangin et al., 1999; Calaforra et al., 2003; Fernández-Cortes et al., 2006) demonstram que este atributo microclimático é o que mais recebe influências diretas da presença humana durante a visitação.

Foram elaborados mapas de superfícies de tendências, de forma a construir isolinhas (isotermas) suavizadas para os gradientes de variação entre ambiente externo e interno das cavernas e possível identificação de impactos de origem antrópica. Também foram gerados mapas de resíduos, no sentido de identificar anomalias locais. Para tanto, seguiu-se a recomendação de Landim (2003) para a distribuição dos pontos de coleta, estipulados ao acaso, mas sem uma concentração maior em uma determinada área. Considerando a irregularidade da distribuição dos pontos de coleta, optou-se pela construção de um reticulado ( $g r i d$ ) para a modelagem da superfície de tendências, procedimento que permite tanto a interpolação quanto a extrapolação dos dados. O reticulado foi processado por regressão polinomial, buscando um ajuste à superfície de maior grau possível (Landim \& Corsi, 2001). Estes procedimentos foram executados com uso do aplicativo Surfer.

Os dados dos atributos microclimáticos foram também cruzados com os valores de $\mathrm{CO}_{2}$ na tentativa de encontrar explicações para as variações encontradas. Estudos feitos em cavernas européias e na Nova Zelândia demonstram a importância de se correlacionar a $\mathrm{T}$ e a UR com as variações de ordem antrópica no
$\mathrm{CO}_{2}$, em função da ativação de processos de corrosão por condensação (Hoyos et al., 1998; Bourges et al., 2001; Freitas \& Schmeckal, 2003). Desta forma, foi feita uma análise de regressão linear múltipla com os dados. Esta foi utilizada para testar a dependência cumulativa de uma variável dependente (y) em relação às diversas variáveis independentes $(\mathrm{x})$, de forma a buscar um comportamento das variáveis " $\mathrm{x}$ " que seja mais útil na explicação - sob o ponto de vista estritamente estatístico - do comportamento da variável "y" (Landim, 2000; 2003). O modelo geral da regressão linear múltipla é representado na equação 3 :

$y_{i}=\mathrm{a}_{0}+\mathrm{a}_{1} x_{1 i}+\ldots+\mathrm{a}_{m} x_{\dot{m}}+\mathrm{e}_{i}$

Sendo que a variância total de "y" é estatisticamente explicada, em parte, pelas diversas variáveis "x", e o restante pela variabilidade devida ao erro $\left(\mathrm{e}_{i}\right)$. Tal explicação não assume um caráter de nexo causal, sendo apenas uma relação numérico-estatística (Landim, 2000; 2003; Lourenço \& Landim, 2004).

A variância de "y" é estatisticamente explicada pelo coeficiente de determinação $\mathrm{R}^{2}$, cujos valores se dispõem no intervalo entre $0-1$. Um coeficiente $\mathrm{R}^{2}$ com valores próximos a 1 representa que as diversas variáveis " $x$ " analisadas são quase que totalmente responsáveis pela variabilidade de "y" (Landim, 2003). Dado que os coeficientes de regressão são parciais, deve-se obter a porcentagem explicada da soma de quadrados de " $\mathrm{y}$ " segundo $2 \mathrm{k}-1$ combinações, tendo " $\mathrm{k}$ " como o número de variáveis independentes (Lourenço \& Landim, 2004).

A regressão linear múltipla foi utilizada para testar estatisticamente duas hipóteses básicas: a) a existência de interferência do evento nos parâmetros ambientais mensurados; e b) identificada uma variável presumidamente alterada pelo evento, esta também foi testada em relação: às demais variáveis, à localização espacial e ao tempo decorrido; buscando investigar também a dinâmica natural do ambiente e sua possível relação com as alterações observadas.

Os testes de regressão linear múltipla e de correlação foram realizados com o aplicativo XL Stat, extensão para análises estatísticas que pode ser anexada ao aplicativo Excel for Windows.

\section{RESULTADOS E DISCUSSÕES}

O total de pessoas simultaneamente dentro da caverna durante o evento musical foi limitado por meio da CCF de Cifuentes. No Salão de Entrada identificouse uma área disponível de $182 \mathrm{~m}^{2}$ para a acomodação das pessoas. No local conhecido como Anfiteatro, face à presença de grande quantidade de blocos abatidos e da própria declividade do terreno, a área disponível foi reduzida a uma área estimada em $56 \mathrm{~m}^{2}$. Para efeito de cálculo, admitiu-se que cada pessoa utiliza $1 \mathrm{~m}^{2}$ de área, de forma a não se sentir desconfortável. Assim, obteve-se o seguinte resultado (equação 4): 
$C C F=\frac{238}{1} * 1$, ou seja, 238 pessoas/evento.

A tabela 1 apresenta o total de pessoas que entraram na caverna, considerando tanto os turistas em visita ao ambiente quanto os expectadores e técnicos presentes durante o evento.

O total de visitas diárias foi superior ao limite apontado, com um excedente de nove visitantes. No entanto, entende-se que a CCF estipulada foi respeitada. Isto porque os 32 visitantes - turistas e monitores ambientais - não se encontravam em visitação dentro da caverna durante o evento, mas sim, distribuídos durante todo o período em que o roteiro estava aberto ao uso público, que vai das 9:00 h às 17:00 h. Entretanto, este total de visitantes não pode ser ignorado para efeitos de identificação de alterações nos parâmetros mensurados, considerando-se a possibilidade de impactos cumulativos no ambiente ao longo do dia.

O monitoramento dos dados de T e UR foi realizado em duas estações externas à caverna e em outras oito em seu interior, especialmente distribuídos em função do Anfiteatro, local onde ocorreu o evento. Os equipamentos das estações 1 (externa, localizado a 22 m da entrada da gruta) e 4 (no Salão de Entrada) apresentaram problemas em seu sensor de medição medida que se adentra a caverna, sugerindo a existência de um gradiente térmico que possa subsidiar o seu zoneamento microclimático.

Outro dado que chama a atenção são os valores máximos e a amplitude, de T e UR, na estação 10 (T: Max.: 21,2 ${ }^{\circ}$ C; Ampl.: 1,8 ${ }^{\circ}$ C; UR: Max.: 96,6\%; Ampl.: 13,5\%). Estes destoam do comportamento médio da caverna, sendo maiores que os da estação 9 (T: Max.: 20,1 ${ }^{\circ}$ C; Ampl.: 0,8 ${ }^{\circ}$ C; UR: Max.: 97,4\%; Ampl.: 10,5\%). - apesar de estarem em um local mais confinado. Sugere-se a possibilidade de que estas variações e máximas sejam decorrentes de fatores antrópicos, já que os picos ocorrem exatamente no início do período pesquisado, quando da instalação dos sensores. Neste caso, tratar-se-ia de um ponto da caverna onde a circulação de energia (cf. classificação de Heaton, 1986) é demasiadamente baixa, carecendo medidas restritivas para visitação dessa parte da caverna. Entretanto, o pequeno período de tempo em que o monitoramento foi realizado e o fato das mesmas alterações não terem sido detectadas durante o momento de retirada dos aparelhos são fatores que lançam dúvidas sobre esta afirmação, de forma a mantê-la como uma hipótese a ser investigada. Esta mesma hipótese pode auxiliar na compreensão da variação da UR na estação 9, com uma amplitude total (10,5\%) superior aos pontos que a antecedem na faixa transicional entre o meio externo e a zona afótica (Estação 2: 19,9\%; 3:

Tabela 1 - Total de pessoas dentro da Gruta do Morro Preto no dia do evento

\section{Local}

Total de pessoas

Salão de Entrada

Anfiteatro (expectadores/técnicos)

Anfiteatro (músicos)

Total parcial - evento

Visitantes (turistas e monitores ambientais) de UR, de tal forma que os dados obtidos não foram utilizados e a comparação entre os dados nas estações internas da gruta tiveram que ser feita apenas com um ponto externo de coleta (estação 2). Os dados coletados foram primeiramente analisados por meio de técnicas de estatística descritiva, considerando um intervalo de confidência de $95 \%$.

Os resultados desta análise evidenciam a existência de uma diferença entre o comportamento microclimático das zonas: externa (estações 1 e 2), salão de entrada (3, 4 e 5) e anfiteatro (6, 7 e 8$)$ - com diferenças mais atenuadas entre ambos - e zona afótica (9 e 10) - figura 2. Na zona externa, a amplitude térmica chegou a $9,8^{\circ} \mathrm{C}$ na estação 1, e a amplitude hígrica a 19,9 \% na estação 2. No caso da $\mathrm{T}$ a amplitude se atenua gradativamente à
4,1\%; 5: 11,3\%; 6: 2,3\%; 7: 4,6\% e 8: 5,8\%) .

A primeira tentativa de explicar a variabilidade nas taxas de $\mathrm{CO}_{2}$ se deu por meio das análises de correlação usando o coeficiente de Pearson. A variável $\mathrm{CO}_{2}$ foi testada em relação às demais variáveis, apresentando os seguintes valores para r: 0,691 (localização dos pontos), 0,481 (realização do evento), 0,250 (temperatura ambiente) e 0,099 (umidade relativa do ar). Apenas as varáveis "localização dos pontos" e "realização do evento" possuem alguma significância de correlação com a variável $\mathrm{CO}_{2}$

Posteriormente, os dados foram trabalhados de forma a apresentar superfícies de tendência para a T. As superfícies de tendência linear e quadrática não apresentaram um ajuste adequado aos dados, 
resultando em isolinhas pouco representativas do ponto de vista de uma provável distribuição dos valores de $\mathrm{T}$ dentro da caverna. Já a superfície cúbica apresentou um ajuste mais adequado - conforme será exposto nos resultados -, sendo determinada pela equação 5:

$$
z(x, y)=A+B x+C y+D x^{2}+E x y+F y^{2}+G x^{3}+H x^{2} y+I x y^{2}+J y^{2}
$$

Onde os pontos de A a $\mathrm{J}$ representam a série de dados de cada uma das estações de monitoramento, de 1 a 10. Desta forma, a figura 3 apresenta dois mapas de isotermas para a Gruta do Morro Preto, um antes do evento e outro após o evento, para fornecer os primeiros subsídios à discussão sobre os impactos antrópicos na temperatura do ar no ambiente da caverna durante o evento.

O que se demonstra é a inexistência de uma relação de impactos na $\mathrm{T}$ ambiente da gruta em função do evento - fato este já evidenciado pelo coeficiente " $r$ " no valor de 0,250 . Os valores de temperatura obtidos nas estações demonstram flutuações para mais e para menos entre os mapas de isotermas 1 e 2, o que está relacionado à dinâmica natural do ambiente. Além disso, há que se considerar a queda de temperatura na estação 1 (externa) de um dia para outro, com uma amplitude térmica de $1,5^{\circ} \mathrm{C}$. Desta forma, as variações detectadas nas demais estações, quando não se tratam dos limites de precisão do equipamento, provavelmente possuem relação direta com a T externa.

Para aprofundar mais os resultados das análises de correlação entre os parâmetros analisados (coeficientes "r"), os valores de T e UR monitorados foram posteriormente cruzados com as seções amostrais de
$\mathrm{CO}_{2}$, considerando o exato minuto e o ponto específico. Com isto, buscou-se averiguar as relações entre as flutuações de $\mathrm{T}$ e UR e as ocorridas no $\mathrm{CO}_{2}$, que foram consideráveis no período mencionado, atingindo máximas de $35,5 \%$ na umidade relativa em relação ao valor inicial na estação 5. Os gráficos de linhas da figura 4 demonstram que os atributos microclimáticos T e UR apresentam variações que oscilam abaixo e acima da média geral obtida, independente de sua relação com o evento. Esta oscilação é diferente para o $\mathrm{CO}_{2}$, que apresenta variações acima da média apenas após o evento..

A figura 4 sugere uma relação direta entre o evento e as taxas de $\mathrm{CO}_{2}$. A média dos valores aferidos na fase pré-evento atingiu o valor de 501,5 ppm. Após o evento, esta média se eleva para 550,61 ppm. Com base nesta constatação foi realizada uma análise de regressão linear múltipla, tendo como variável dependente a realização do evento. As variáveis independentes foram os valores de $\mathrm{T}$, UR e $\mathrm{CO}_{2}$. Os dados foram analisados por meio do método step wise e uma margem de erro máxima aceitável de 5\%. As variáveis independentes foram confrontadas com a dependente uma a uma, depois aos pares e por fim, todas juntas. Considerando todas as variáveis, obteve-se como resultados a equação 6 e os seguintes coeficientes $\mathrm{R}^{2}$ : $\mathrm{CO}_{2}$ : 0,232; UR: 0,055; T: 0,002; $\mathrm{CO}_{2}+\mathrm{T}+\mathrm{UR}: 0,232$.

Evento $=-3,68869365074147+, 25525268799529 \mathrm{E}-$ $03 * \mathrm{CO}_{2}$; com $\mathrm{R}^{2}=0,232$.

Os valores $\mathrm{R}^{2}$ obtidos evidenciam a baixa interferência da variável "evento" nas variáveis T e

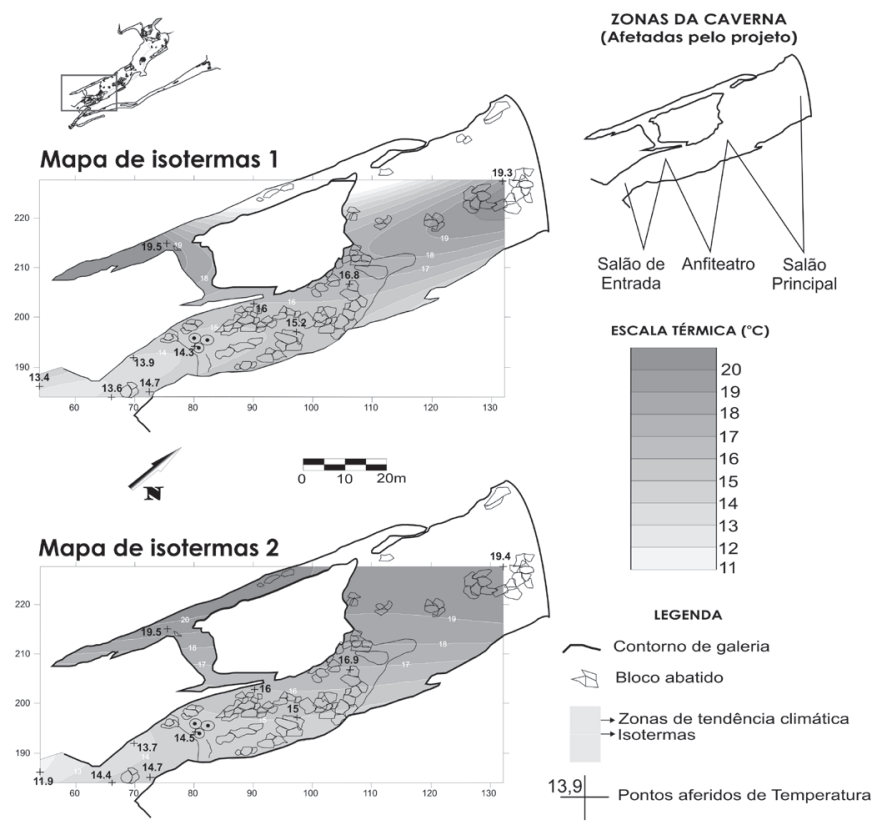

Figura 3 - Superfícies de tendência de terceira ordem para o microclima da Gruta do Morro Preto, considerando duas situações: Mapa de isotermas 1 - antes do evento, às 9:00 hs do dia 17/05/2008;

Mapa de isotermas 2 - aproximadamente 18 hs após o evento, às 9:00 hs do dia 18/05/2008.

$O$ evento musical teve início às 15:00 hs e encerramento às 16:00 h do dia 17/05/2008.

Elaborada pelos autores, sob planta original de GEGEO (1998). 


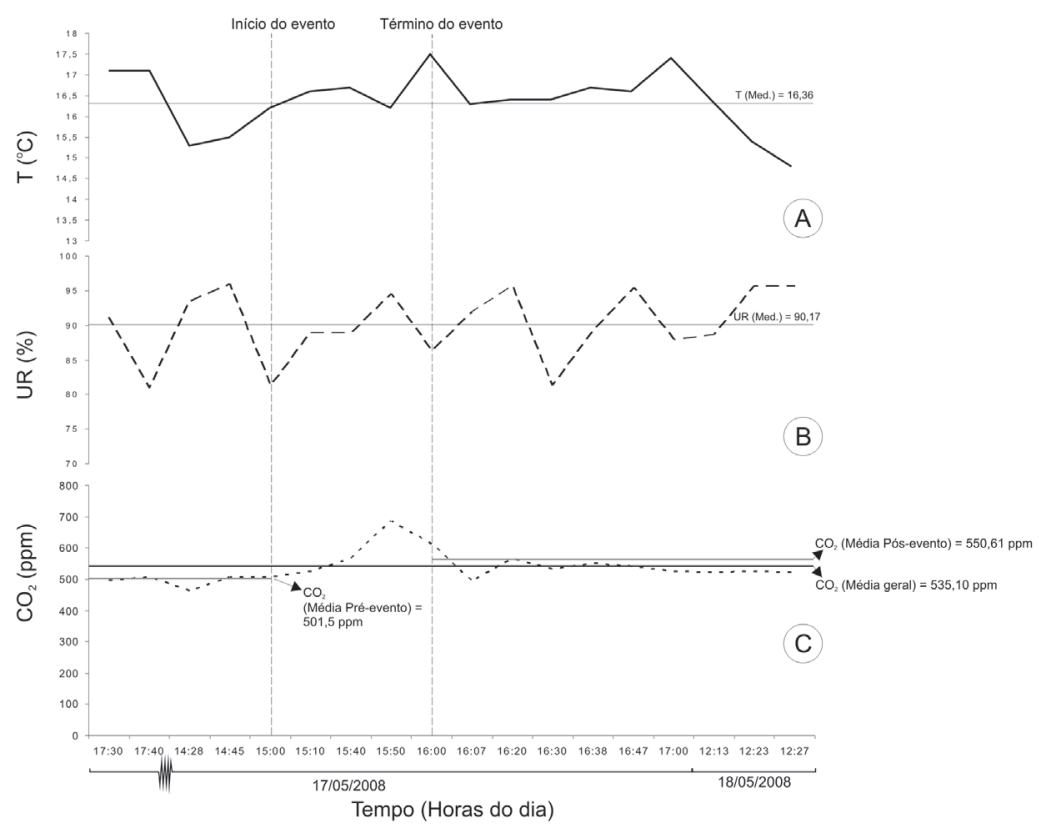

Figura 4 - Comparação entre as variáveis mensuradas em relação ao evento. As linhas verticais pontilhadas representam o momento de início e término do evento na caverna. Muito embora as variáveis UR e T possuam valores acima da média (4A e 4B), note-se que estes não possuem relação direta com o evento, já que os picos aparecem nos gráficos em diversos momentos. Por sua vez, o gráfico $4 C$, correspondente ao $\mathrm{CO}_{2}$, sugere uma relação de alteração na variável em função do evento, dado que os valores acima da média aparecem somente durante e após a sua realização.

UR, tal qual já observado pelo coeficiente "r", dado que nos confrontos aos pares e com todas as variáveis, prevalecem os valores relativos à variável independente $\mathrm{CO}_{2}\left(\mathrm{R}^{2}=0,232\right.$, ou $\left.23,2 \%\right)$. As variáveis UR e $\mathrm{T}$ aparecem somente na ausência do $\mathrm{CO}_{2}$ na análise, e ainda assim em percentuais bem menos significativos (com coeficientes $\mathrm{R}^{2}=0,055$ ou $5,5 \%$ para UR e 0,002 ou $0,2 \%$ para $\mathrm{T}$ ).

Este resultado suscita algumas preocupações, dado que estudos como o de Hoyos et al. (1998) demonstram que o aumento nas taxas de $\mathrm{CO}_{2}$ no ambiente cavernícola pode causar danos no meio físico. Isto porque o aumento na concentração deste gás pode tornar a água resultante do processo de percolação novamente agressiva, ao gerar o ácido carbônico. Com isso, a solução voltaria a dissolver os espeleotemas e/ou ampliar/ativar processos de corrosão por condensação nas paredes e teto da caverna.

Por outro lado, é preciso considerar a possibilidade de intervenção da dinâmica natural do sistema no resultado. Para tanto, a variável $\mathrm{CO}_{2}$ foi também testada como dependente, tendo como variáveis independentes: T, UR, evento, localização espacial dos pontos e variação temporal. Considerando os mesmos métodos e margem de erro, obteve-se a equação 7 e a análise de variância apresentada na tabela 2 :

$\mathrm{CO}_{2}=-187,113469829281+7,5139970675973 * \mathrm{Am}-$ biente $+6,59291679700539 * \mathrm{UR}$; com um coeficiente de determinação $\mathrm{R}^{2}=0,805$.
A análise de variância demonstra que pelo menos duas das variáveis dependentes explicam em aproximadamente $80 \%$ a variabilidade de $\mathrm{CO}_{2}$. O teste $\mathrm{F}$ indica que estas variáveis reduzem significativamente a variação da variável dependente. A equação obtida destaca as variáveis independentes "localização espacial dos pontos de coleta" e "UR", que respondem sozinhas pelo percentual obtido de variância na variável dependente $\mathrm{CO}_{2}$.

Para identificar a contribuição pura de cada variável na predição da variabilidade de $\mathrm{CO}_{2}$, as variáveis independentes foram testadas em etapas individuais e combinadas aos pares, trios, quartetos e com todas em conjunto, gerando um total de 29 combinações distintas. Dentre estas, foram selecionadas aquelas com maior coeficiente $\mathrm{R}^{2}$ em confronto com a variável dependente, apresentados na tabela 3 .

Os resultados das tabelas 2 e 3 e da equação 7 evidenciam que, dentro dos parâmetros selecionados, apenas as variáveis "ambiente de coleta" e "UR" possuem relação direta com a variabilidade de $\mathrm{CO}_{2}$. As demais variáveis passam a apresentar alguma significância apenas com o aumento da margem de erro aceitável para o teste estatístico, elevando-o para 40\%, mas ainda assim com contribuições ínfimas: $3,1 \%$ para evento; 3,6\% para Tempo e 1\% para T. Entretanto, estes valores são considerados apenas a título de ordenamento hierárquico das variáveis, já que a margem de erro adotada lança dúvidas sobre qualquer conclusão que se pretenda obter considerando os objetivos da pesquisa 
Tabela 2 - Análise de variância em relação à variável dependente $\mathrm{CO}_{2}$

\begin{tabular}{|c|c|c|c|c|}
\hline Fonte de variação & g.l. & Soma dos Quadrados & Médias Quadráticas & Razão F \\
\hline Modelo & 2 & 33985,200 & 16992,600 & 30,877 \\
\hline Resíduos & 15 & 8255,078 & 550,339 & \\
\hline Total & 17 & 42240,278 & & \\
\hline
\end{tabular}

realizada.

Os resultados da tabela 3 vão ao encontro de estudos anteriores sobre a variação nas taxas de $\mathrm{CO}_{2}$ no ambiente cavernícola. Linhua et al. (2000), em pesquisas na caverna Bayun, China, demonstraram que as concentrações de $\mathrm{CO}_{2}$ no ambiente em função da visitação aumentaram à medida que se avança em seu interior. Os autores também comprovaram que, em espaços mais confinados, a presença humana causa alterações significativas nas taxas de $\mathrm{CO}_{2}$. Outros trabalhos, como os de Hoyos et al. (1998), Fernández- concentração de $\mathrm{CO}_{2}$ no ambiente. As pesquisas feitas por Fernández-Cortes et al. (2006) e Liñán et al. (2008) demonstraram existir uma forte relação entre este parâmetro e as estações do ano. Assim, o presente estudo deve ser ampliado, para que outras hipóteses venham a ser testadas em um horizonte temporal mais amplo.

Além dos aspectos investigados, outros devem ser considerados para ampliar novos estudos sobre o tema e balizar a investigação de hipóteses pautadas na mesma linha de estudos, que relaciona a presença humana em

Tabela 3 - Contribuição pura das variáveis independentes em relação à variabilidade do $\mathrm{CO}_{2}$

\begin{tabular}{lccc}
\hline \multicolumn{1}{c}{ Etapa (resultados mais significativos) } & $\mathrm{R}^{2}$ & Variável & Contribuição \\
\hline & & & percentual \\
I - Ambiente & 0,686 & Ambiente & $68,6 \%$ \\
II - Ambiente + UR & 0,805 & UR & $11,9 \%$ \\
III - Ambiente + UR + Evento & 0,805 & Evento & - \\
IV -T + Ambiente + UR + Evento & 0,805 & T & - \\
V - T + Ambiente + UR + Evento + & 0,805 & Tempo & - \\
Tempo & & & \\
\hline
\end{tabular}

Cortes et al. (2006) e Liñán et al. (2008) evidenciam as relações entre o confinamento espacial das cavernas e sua distância em relação às entradas com a concentração de $\mathrm{CO}_{2}$. Tais resultados foram também evidenciados nesta pesquisa.

Entretanto, não se descartam aqui os resultados evidenciados por meio dos coeficientes $r$ e $R^{2}$ e da figura 5, que demonstram a existência de uma relação estatística entre a variabilidade de $\mathrm{CO}_{2}$ e a realização do evento. Desta forma, percebe-se que tanto a localização dos pontos de coleta - o ambiente - quanto o evento são importantes para explicar a variabilidade nas taxas de $\mathrm{CO}_{2}$.

Assim sendo, é preciso considerar a necessidade de um estudo sazonal mais aprofundado na gruta do Morro Preto, de forma a identificar padrões de concentração de $\mathrm{CO}_{2}$ em função das estações seca/chuvosa e da amplitude térmica. A presente pesquisa evidenciou a importância da UR - parâmetro derivado da T e da umidade absoluta do ar, que por sua vez depende da disponibilidade hígrica, ou seja, das chuvas - na cavernas a alterações em seus parâmetros ambientais:

Microclima externo: as mudanças climáticas externas provavelmente interferiram nos valores identificados. Nos dias 16 e 17 de Maio de 2008 o clima encontrava-se estável, com céu aberto. No dia seguinte houve uma repentina alteração, com o aumento da UR em função da chuva. Ao que se constatou por meio das análises estatísticas (coeficiente $\mathrm{R}^{2}$ ) e de estudos anteriores (Fernández-Cortes et al., 2006; Liñán et al., 2008), o comportamento hígrico do ambiente interfere nas concentrações de $\mathrm{CO}_{2}$

Antrópicos (diretos ou indiretos): o aumento geral nos níveis de $\mathrm{CO}_{2}$ pode estar relacionado à dispersão do gás gerado pela respiração das pessoas no dia anterior, durante o evento; a presença de pessoas dentro da caverna pode ter provocado a remobilização da matéria orgânica disponível no solo, pelo pisoteio, o que acarretaria na aceleração metabólica de microorganismos ali existentes com elevação do teor de $\mathrm{CO}_{2}$ no interior da caverna. 


\section{CONCLUSÕES}

Em relação aos diversos aspectos observados durante o experimento de monitoramento de um evento musical na Gruta do Morro Preto, foram obtidas as seguintes conclusões:

Relação evento versus T e UR: O evento realizado não trouxe interferências significativas para os parâmetros de T e UR, conforme as análises estatísticas realizadas. Este resultado é significativo, dado que estudos diversos (e.g. Pulido-Bosch et al., 1997; Hoyos et al., 1998; Mangin et al., 1999; Calaforra et al., 2003; Fernández-Cortés et al., 2006; Lima \& Morais, 2006; Boggiani et al., 2007) ressaltam a importância destes atributos microclimáticos para o manejo espeleológico

Relação evento versus $\mathrm{CO}_{2}$ : A análise de regressão linear e o coeficiente de relação produto-momento de Pearson demonstraram que dentre as variáveis analisadas, apenas as taxas de $\mathrm{CO}_{2}$ receberam alterações em função do evento. Ao analisar o parâmetro em questão em relação à dinâmica climática e considerando também fatores espaciais, o mesmo procedimento estatístico evidenciou alterações em função dos pontos de coleta de dados e da UR. Se por um lado a hipótese de impacto atmosférico devido à concentração de pessoas não pôde ser confirmada, por outro lado tampouco pode ser descartada. Novos estudos em diferentes épocas do ano se fazem necessários para identificar até que ponto as alterações detectadas decorrem da dinâmica natural do sistema e a partir de quando podem ser consideradas como impactos ambientais de origem antrópica

Desta forma, pode-se concluir que eventos musicais controlados e com características semelhantes ao realizado não causam danos ambientais na gruta do Morro Preto, se limitando, na pior das hipóteses, a um nível irrelevante de impactos nas concentrações de $\mathrm{CO}_{2}$. Todavia, estudos ligados aos impactos no solo e na fauna, hipoteticamente causados, respectivamente, pelo pisoteamento desordenado e por distúrbios sonoros, devem ser realizados para ampliar esta perspectiva de análise e fornecer subsídios para um manejo mais adequado da cavidade natural em questão.

\section{AGRADECIMENTOS}

Os autores agradecem as seguintes pessoas e instituições, que de diversas formas contribuíram para o estudo realizado: Sra. Kátia Pisciotta - Fundação Florestal-SMA/SP; Sr. Fábio Leonardo Tomas - Parque Estadual Turístico do Alto Ribeira - PETAR, SP; Sra. Maria Margareth Escobar Ribas Lima-Superintendente da Regional Mato Grosso do Sul do Instituto do Patrimônio Histórico e Artístico Nacional - IPHAN -, pelo empréstimo dos termohigrômetros; Profa. Dra. Dejanira Franceschi de Angelis - Departamento de Bioquímica, Instituto de Biociências - IB/UNESP - Rio Claro, SP, pelo empréstimo do medidor de $\mathrm{CO}_{2}$.

\section{REFERÊNCIAS}

BOGGIANI, P.C., SILVA, O.J. DA, GESICKI, A.L.D., GALLATI, E.A.B., SALLES, L. DE O., LIMA, M.M.E.R. 2007. Definição de capacidade de carga turística das cavernas do Monumento Natural Gruta do Lago Azul (Bonito,MS). Geociências, 26:333348.

BOURGES, F., MANGIN, A., D'HULST, D. 2001. Le gaz carbonique dans la dynamique de l'atmosphère des cavités karstiques: l'exemple de l'Aven d'Orgnac (Ardèche). Sciences de la Terra et des Planètes, 333:685-692.

CALAFORRA, J.M., FERNÁNDEZ-CORTÉS, A., SÁNCHESMARTOS, F., GISBERT, J., PULIDO-BOSCH, A. 2003. Environmental control for determining human impact and permanent visitor capacity in a potential show cave before tourist use. Environmental Conservation, 30:160-167.

CAMPANHA, G.A.C. 1991. Tectônica neoproterozóica no alto e médio Vale do Ribeira, estados de São Paulo e Paraná. Tese de Doutorado, Instituto de Geociências, Universidade de São Paulo. 296 p.

CIFUENTES-ARIAS, M., MESQUiTA, C.A.B., MÉNDEZ, J., MORALES, M.E., AGUILAR, N., CANCINO, D., GALLO, M., RAMIREZ, C., RIBEIRO, N., SANDOVAL, E., TURCIOS, M. 1999. Capacidad de carga turística de las áreas de uso público del Monumento Nacional Guayabo, Costa Rica. CATIE/WWF, Turrialba, $75 \mathrm{pp}$.

CIGNA, A.A. \& FORTI, P. 1988. The environmental impact assessment of a tourist cave. In: UIS, Cave Tourism International Symposium At-170 Anniversary of Postojnska Jama, Postojna (Yugoslavia), Proceedings, p. 29-38.

FERNÁNDEZ-CORTÉS, A., CALAFORRA, J.M., SÁNCHEZMARTOS, F. 2006. Spatiotemporal analysis of air conditions as a tool for the environmental management of a show cave (Cueva del Agua, Spain). Atmospheric Environment, 40:7378-7394.

FREITAS, C.R. DE \& SCHMEKAL, A. 2003. Condensation as a microclimate process: Measurement, numerical simulation and prediction in the Glowworm Cave, New Zealand. International Journal of Climatology, 23:557-575.

GERARDI, L.H. DE O. \& SILVA, B.C.N. 1981. Quantificação em geografia. Difel, São Paulo, 161 pp.

GEGEO - GRUPO DA GEO DE ESPELEOLOGIA. 1998. Conjunto Morro Preto / Couto. USP/Gegeo, São Paulo, 1 p.

HEATON, T. 1986. Caves: a tremendous range in energy environments on earth. National Speleological Society News, 8:301-304.

HOYOS, M., SOLER, V., CAÑAVERAS, J.C., SÁNCHEZ-MORAL, S., SANZ-RUBIO, E. 1998. Microclimatic characterization of a karstic cave: human impact on microenvironmental parameters of a prehistoric rock art cave (Candamo Cave, Northern Spain). Environmental Geology, 33:231-242.

KARMANN, I. \& FERRARI, J.A. 2002. CARSTE E CAVERNAS DO PARQUE ESTADUAL TURÍSTICO DO ALTO RIBEIRA (PETAR), SP. IN: WINGE, M., SCHOBBENHAUS, C., SOUZA, C.R.G., FERNANDES, A.C.S., BERBERT-BORN, M., QUEIROZ, E.T. (EDS.) Sítios geológicos e paleontológicos do Brasil. DNPM, Brasília, pp. 401-413.

LANDIM, P.M.B. 2000. Análise estatística de dados geológicos multivariados. IGCE/DGA, Rio Claro, $128 \mathrm{pp}$.

LANDIM, P.M.B. 2003. Análise estatística de dados geológicos. 2.ed. Edunesp, Rio Claro, 253 pp.

LANDIM, P.M.B. \& CORSI, A.C. 2001. Cálculo de superfícies de tendência, por regressão polinomial, pelo SURFER. IGCE/ DGA, Rio Claro, 11 pp.

LIMA, T.F. \& MORAIS, M.S. DE. 2006. Contribuições para o desenvolvimento de plano de manejo em ambiente cavernícola - gruta do Maquine: um estudo de caso. Geonomos, 14:45-53.

LIÑÁN, C., VADILLO, I., CARRASCO, F. 2008. Carbon dioxide concentration in air within the Nerja cave (Malaga, Andalusia, Spain). International Journal of Speleology, 37:99-106.

LINHUA, S.; XIAONING, W.; FUYUAN, L. 2000. The influences of cave tourism on $\mathrm{CO}_{2}$ and temperature in Baiyun cave, Hebei, China. International Journal of Speleology, 29B:77-87.

LOBO, H.A.S. 2008. Ecoturismo e percepção de impactos socioambientais sob a ótica dos turistas no Parque Estadual Turístico do Alto Ribeira - PETAR. Pesquisas em Turismo e 
Paisagens Cársticas, 1:67-76.

LONGHITANO, G.A., ROCHA, B.N., ÂNGELO FURLAN, S. Caracterização microclimática da gruta Colorida - Parque Estadual de Intervales, SP. In: SEE/SBE, Cong. Bras. Espeleologia, 29, Anais, p. 1-7.

LOURENÇO, R.W. \& LANDIM, P.M.B. 2004. Análise de regressão múltipla espacial. IGCE/DGA/Lab. Geomatemática, Rio Claro, $34 \mathrm{pp}$.
MANGIN, A., BOURGES, F., HULST, D. 1999. La conservation des grottes ornées: Un problème de stabilité d'um système naturel (l'exemple de la grotte préhistorique de Gargas, Pyrénées Françaises). Sciences de la Terra et des Planètes, 328:295-301.

PULIDO-BOSCH, A., MARTÍN-ROSALES, W., LÓPEZCHICANO, M., RODRÍGUEZ-NAVARRO, M., VAllejos, A. 1997. Human impact in a tourist karstic cave (Aracena, Spain). Environmental Geology, 31:142-149. 